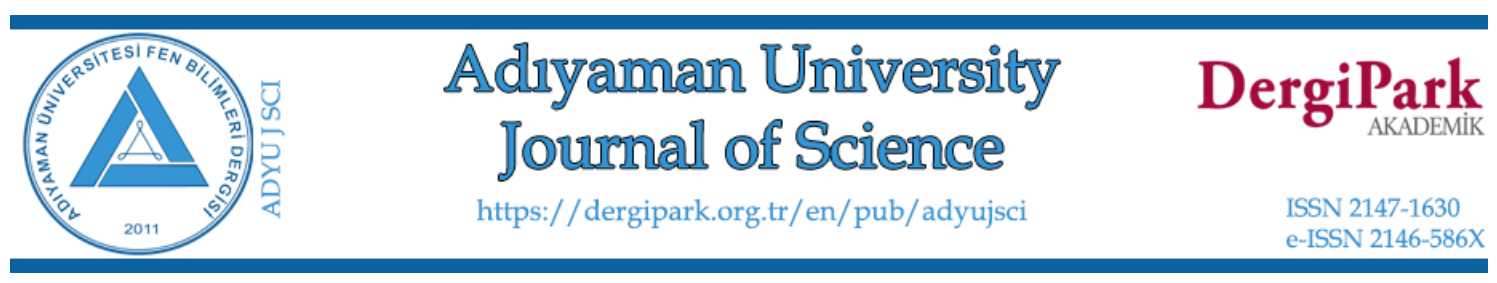

\title{
Soft Quasilinear Spaces and Soft Normed Quasilinear Spaces
}

\author{
Hacer BOZKURT ${ }^{1, *}$ \\ ${ }^{1}$ Batman University, Faculty of Science and Letters, Department of Mathematics, Batman, Turkey \\ hacer.bozkurt@batman.edu.trORCID:0000-0002-2216-2516
}

\begin{tabular}{lll}
\hline Received: 29.04.2020 & Accepted: 09.11.2020 & Published: 30.12.2020
\end{tabular}

\begin{abstract}
In this study, a recent concepts of soft quasilinear spaces and soft proper quasilinear spaces are presented. Further, soft quasi vectors in soft quasilinear spaces are investigated, and several related properties are examined such as quasilinear dependent and quasilinear independent. Also, the concept of soft quasi norm of soft quasilinear spaces is given. Lastly, soft quasilinear operators on soft normed quasilinear spaces are defined, and some results about the bounded soft quasilinear operators and continuous soft quasilinear operators are obtained.
\end{abstract}

Keywords: Soft quasilinear space; Soft quasi vector; Soft normed quasilinear space; Soft quasilinear operator.

\section{Esnek Quasilineer Uzaylar ve Esnek Normlu Quasilineer Uzaylar}

\section{Öz}

$\mathrm{Bu}$ çalışmada, yeni bir kavram olan esnek quasilineer uzay ve esnek proper quasilineer uzay kavramları sunulmuştur. Ayrıca esnek quasilineer uzayda bir esnek quasi vektör tanımı verilmiş ve bu yeni kavram ile ilgili quasilineer bağımlılık-bağımsızlık özellikleri ele alınmıştır. Esnek quasilineer uzaylarda esnek quasi norm kavramı tanıtılmıştır. Son olarak bir esnek normlu quasilineer uzayda esnek quasilineer operatör tanımı verilmiş ve sınırlı esnek quasilineer operatör ile sürekli esnek quasilineer operatörlerle ilgili bazı sonuçlar elde edilmiştir. 
Anahtar Kelimeler: Esnek quasilineer uzay; Esnek quasi vektör; Esnek normlu quasilineer uzay; Esnek quasilineer operatör.

\section{Introduction}

In [1], Molodtsov introduced the notion of soft sets which is an unexplored mathematical tool for dealing with ambiguities. After, many papers regarding soft sets were published. In the paper [2] and [3], the authors preferred the notion of soft element by handling a function and they investigated soft real number by using soft element, respectively. After then, in [2], soft vector space was presented by handling the notion of soft element and in $[4,5]$ they worked on soft normed spaces and soft linear operators and their fundamental properties.

On the other hand, in the paper [6], Aseev offered the notions of quasilinear spaces and normed quasilinear spaces. Owing to these new definitions, he obtained some results consistent with linear spaces. In [7], they presented the idea of topological quasilinear spaces. In [8], they worked on proper quasilinear spaces and explored quasilinear dependence-independence. In [9], they worked on inner product quasilinear spaces. After, in $[10,11]$, the authors studied on some properties of quasilinear spaces.

Actually, in the existing paper, we define the concepts of soft quasilinear spaces, soft normed quasilinear spaces and soft quasilinear operators. In section two, we give some preliminaries about the soft sets and quasilinear spaces. In section three, we give the soft quasilinear spaces as a new concept and search some of its properties with examples. Further, we study soft proper quasilinear spaces. In section four, we define the soft norm in soft quasilinear spaces and present soft quasilnear operators and its various properties.

\section{Preliminaries}

Firstly, we remember some notions in soft set theory and some basic concepts such as quasilinear spaces and normed quasilinear spaces.

Let $V$ be an universe and $F$ be a set of all probable parameters. Let $P(V)$ indicate the power set of $V$ and $B$ be a non-empty subset of $F$.

Definition 1. [1] A pair $(G, B)$ is called a soft set over $V$, where $G$ is a mapping defined by $G: B \rightarrow P(V)$.

Definition 2. [12] A soft set $(G, B)$ over $V$ is said to be a null soft set represented by $\Phi$, if for every $b \in B, G(b)=\emptyset$. 
Definition 3. [13] For a soft set $(G, B)$ over $V$, the set $\operatorname{Supp}(G, B)=\{b \in B: G(b) \neq \emptyset\}$ is called support of the soft set $(G, B)$. The null soft set is a soft set with an empty support. A soft set $(G, B)$ is non-null if $\operatorname{Supp}(G, B) \neq \varnothing$.

Definition 4. [12] A soft set $(G, B)$ over $V$ is said to be an absolute soft set represented by $\tilde{V}$, if for every $b \in B, G(b)=V$.

Definition 5. [3] Let $V \neq \varnothing$ and $B$ be a nonempty parameter set. Then a function $\varepsilon: B \rightarrow V$ is said to be soft element of $V$.

Definition 6. $[14,15]$ A soft set $(G, B)$ over $V$ is said to be a soft point if there is certainly a $b \in B$, such that $G(b)=\{v\}$ for some $v \in V$ and $G\left(b^{\prime}\right)=\varnothing$, for every $b^{\prime} \in V /\{b\}$. It will be indicated by $\tilde{v}_{b}$.

Definition 7. [6] A quasilinear space over a field $\mathbb{R}$ is a set $Q$ with a partial order relation $\preccurlyeq$, with the operations of addition $Q \times Q \rightarrow Q$ and scalar multiplication $\mathbb{R} \times Q \rightarrow Q$ satisfying the following conditions:

$(Q 1) q \preccurlyeq q$,

(Q2) $q \preccurlyeq z$, if $q \preccurlyeq w$ and $w \preccurlyeq z$,

(Q3) $q=w$, if $q \preccurlyeq w$ and $w \preccurlyeq q$,

$(Q 4) q+w=w+q$,

$(Q 5) q+(w+z)=(q+w)+z$

(Q6) there exists an element $\theta \in Q$ such that $q+\theta=q$,

$(Q 7) \alpha \cdot(\beta \cdot q)=(\alpha \cdot \beta) \cdot q$,

(Q8) $\alpha \cdot(q+w)=\alpha \cdot q+\alpha \cdot w$

(Q9) $1 \cdot q=q$,

$(Q 10) 0 \cdot q=\theta$

$(Q 11)(\alpha+\beta) \cdot q \preccurlyeq \alpha \cdot q+\beta \cdot q$,

$(Q 12) q+z \preccurlyeq w+v$, if $q \preccurlyeq w$ and $z \preccurlyeq v$,

(Q13) $\alpha \cdot q \preccurlyeq \alpha \cdot w$, if $q \preccurlyeq w$, 
for every $q, w, z, v \in Q$ and every $\alpha, \beta \in \mathbb{R}$.

If an element $q$ has an inverse, then it is called regular. If an element $q$ has no inverse, then it is called singular. Also, $Q_{r}$ express for the set of all regular elements in $Q$ and $Q_{s}$ imply the sets of all singular elements in $Q$. Besides, $Q_{r}, Q_{d}$ and $Q_{s} \cup\{0\}$ are subspaces of $Q$, where $Q_{r}$ regular subspace of $Q, Q_{d}$ symmetric subspace of $Q$ and $Q_{s} \cup\{0\}$ singular subspace of $Q$. Further, let $Q$ be a quasilinear space, $W \subseteq Q$ and $q \in W$. The set

$$
F_{q}^{W}=\left\{m \in W_{r}: m \preccurlyeq q\right\}
$$

is called floor in $W$ of $q$. In the case of $W=Q$ it is called only floor of $q$ and written briefly $F_{q}$ instead of $F_{q}^{Q}[8]$.

Definition 8. [8] Let $Q$ be a quasilinear space, $W \subseteq Q$ and $q, w \in W$. $W$ is called proper set if the following cases hold:

$(P Q 1) F_{q}^{W} \neq \varnothing$ for every $q \in W$

$(P Q 2) F_{q}^{W} \neq F_{w}^{W}$ for all pair of points $q, w$ such that $q \neq w$.

Definition 9. [6] Let $Q$ be a quasilinear space. A function $\|.\|_{Q}: Q \rightarrow \mathbb{R}$ is named a norm if the following circumstances hold:

$$
\begin{aligned}
& (N Q 1)\|q\|_{Q}>0 \text { if } q \neq 0, \\
& (N Q 2)\|q+w\|_{Q} \leq\|q\|_{Q}+\|w\|_{Q}, \\
& (N Q 3)\|\alpha \cdot q\|_{Q}=|\alpha| \cdot\|q\|_{Q}, \\
& (N Q 4) \text { if } q \leqslant w, \text { then }\|q\|_{Q} \leq\|w\|_{Q},
\end{aligned}
$$

$(N Q 5)$ if for any $\varepsilon>0$ there exists an element $q_{\varepsilon} \in Q$ such that, $q \preccurlyeq w+q_{\varepsilon}$ and $\left\|q_{\varepsilon}\right\|_{Q} \leq$ $\varepsilon$ then $q \preccurlyeq w$ for any elements $q, w \in Q$ and any real number $\alpha \in \mathbb{R}$.

A quasilinear space $Q$ is called normed quasilinear space with a norm defined on it. Let $Q$ be a normed quasilinear space. Then, Hausdorff or norm metric on $Q$ is defined by

$$
h_{Q}(q, w)=\inf \left\{r \geq 0: q \preccurlyeq w+a_{1}^{r}, w \preccurlyeq q+a_{2}^{r},\left\|a_{i}^{r}\right\| \leq r\right\} .
$$

Definition 10. [6] Let $Q$ and $W$ be quasilinear spaces. Then a quasilinear operator $\lambda: Q \rightarrow$ $W$ is a function satisfying: 
$(Q 01) \lambda(\alpha \cdot q)=\alpha \cdot \lambda(q)$,

$(Q 02) \lambda(q+w) \preccurlyeq \lambda(q)+\lambda(w)$

$(Q 03) \lambda(q) \preccurlyeq \lambda(w)$, if $q \preccurlyeq w$ for any $q, w \in Q$ and $\alpha \in \mathbb{R}$.

\section{Soft Quasilinear Spaces}

In this part, we present the definitions of soft quasilinear space and soft proper quasilinear space which are given the first time.

Let $Q$ be a quasilinear space over $\mathbb{R}$ and $B$ be a parameter set. If we define a function $G: B \rightarrow P(Q)$ such that $G(b)=P(y) \in Q$ for every $b \in B$, then $(G, B)$ is called a soft set over $Q$.

Definition 11. Let $(G, B)$ be a non-null soft set over a quasilinear space $Q$. Then $(G, B)$ is called a soft quasilinear space over $Q$ if $G(b)$ is a subquasilinear space of $Q$ for every $b \in$ $\operatorname{Supp}(G, B)$.

Example 12. Let $Q=\Omega_{C}(\mathbb{R})$ be the quasilinear space and $\left(G,\left(\Omega_{C}(\mathbb{R})\right)_{r}\right)$ be a soft set over $Q$ and $G:\left(\Omega_{C}(\mathbb{R})\right)_{r} \rightarrow P(Q)$ is a function described by

$$
G(X)=\left\{[-X, X]: X \in\left(\Omega_{C}(\mathbb{R})\right)_{r}\right\}
$$

Since $G(X)$ is a subquasilinear space of $\Omega_{C}(\mathbb{R})$ for every $X \in \operatorname{Supp}\left(G,\left(\Omega_{C}(\mathbb{R})\right)_{r}\right)$, $\left(G,\left(\Omega_{C}(\mathbb{R})\right)_{r}\right)$ is called a soft quasilinear space over $\Omega_{C}(\mathbb{R})$.

Example 13. Consider the intervals quasilinear space $I \mathbb{R}^{2}$ over $\mathbb{R}$ and $(G, B)$ be a soft set over $I \mathbb{R}^{2}$, where $B=I \mathbb{R}$ and $G: I \mathbb{R} \rightarrow P\left(I \mathbb{R}^{2}\right)$ be defined as follows:

$$
G(X)=\{(X \cdot r, 0): r \in \mathbb{R}\},
$$

for every $X \in I \mathbb{R}$. Then $G(X)$ is a subquasilinear space of $I \mathbb{R}^{2}$ for every $X \in \operatorname{Supp}(G, I \mathbb{R})$. Then $(G, I \mathbb{R})$ is a soft quasilinear space of $I \mathbb{R}^{2}$. If we define another function $F: I \mathbb{R} \rightarrow I \mathbb{R}^{2}$ such that

$$
F(X)=\{(X \cdot r, 1): r \in \mathbb{R}\}
$$

for every $(X) \in I \mathbb{R}$. Then $F$ is a not soft quasilinear space of $I \mathbb{R}^{2}$.

If every element in soft quasilinear space $(G, B)$ has an reverse element in $(G, B)$ then notion of soft quasilinear space coincides with the soft linear space. 
Definition 14. Assume that $(G, B)$ is a soft quasilinear space of $Q$ over $\mathbb{R}$ and $(F, B) \subseteq$ $(G, B)$ is a soft set over $(G, B)$. Then $(F, B)$ is called a soft subquasilinear space of $(G, B)$ whenever $(F, B)$ is quasilinear space with identical partial ordering and identical operations on $Q$.

Theorem 15. A soft subset $(F, B)$ of a soft quasilinear space $(G, B)$ is a soft subquasilinear space of a soft quasilinear space $(G, B)$ if and only if $\alpha \cdot F+\beta \cdot F \subset F$ for every $\alpha, \beta \in \mathbb{R}$.

Proof. Let $(F, B)$ be a soft subquasilinear space of a soft quasilinear space $(G, B)$ of $Q$ over $\mathbb{R}$ and $b \in B$. Then

$$
(\alpha \cdot F+\beta \cdot F)(b) \subset F(b),
$$

since $F(b)$ is a subquasilinear space and $\alpha \cdot M+\beta \cdot N \in F(b)$ for every $M, N \in F(b)$ and $\alpha, \beta \in$ $\mathbb{R}$.

Let $\alpha \cdot F+\beta \cdot F \subset F$ for all scalars $\alpha, \beta \in \mathbb{R}$. Then

$$
(\alpha \cdot F+\beta \cdot F)(b)=\{\alpha \cdot M+\beta \cdot N: M, N \in F(b)\} \subset F(b)
$$

for every $\alpha, \beta \in \mathbb{R}$ and for all $b \in B$. From here, we get $\alpha \cdot M+\beta \cdot N \in F(b)$ for all $M, N \in F(b)$ and $\alpha, \beta \in \mathbb{R}$. Hence, $F(b)$ is a subquasilinear space of $Q$ over $\mathbb{R}$ for all $b \in B$. Since $(F, B)$ is a soft subset of $(G, B), F(b) \subset G(b)$ for every $b \in B$. Thus, $(F, B)$ is a soft subquasilinear space of $(G, B)$.

Definition 16. Let $(G, B)$ be a soft quasilinear space over $Q$ and $(F, C)$ be a soft quasilinear space over $P$. The product of soft quasilinear spaces $(G, B)$ and $(F, C)$ is described as $(G, B) \times(F, C)=(V, B \times C)$, where $V(b, c)=G(b) \times F(c)$ for every $(b, c) \in B \times C$.

Theorem 17. Let $(G, B)$ be a soft quasilinear space over $Q$ and $(F, C)$ be a soft quasilinear space over $P$. If it is non-null, then $(G, B) \times(F, C)$ is a soft quasilinear space over $Q \times P$.

Proof. Proof is similar to soft linear spaces.

Definition 18. Let $(G, B)$ and $(F, C)$ be two soft quasilinear spaces over $Q$. The algebraic sum operation of soft quasilinear spaces $(G, B)$ and $(F, C)$ is defined as $(G, B)+(F, C)=$ $(E, B \times C)$, where

$$
E(b, c)=G(b)+F(c)
$$

for every $(b, c) \in B \times C$. 
Theorem 19. Let $(G, B)$ and $(F, C)$ be two soft quasilinear spaces over $Q$. If it is non-null, then $(G, B)+(F, C)$ and $\alpha \cdot(G, B)$ are soft quasilinear spaces over $Q$.

Proof. Proof is similar to soft linear spaces.

Example 20. Let $Q=M_{2}\left(\Omega_{C}(\mathbb{R})\right)$ be the quasilinear space over $\mathbb{R}, B=2 \cdot \Omega_{C}(\mathbb{R}), C=$ $4 \cdot \Omega_{C}(\mathbb{R})$. Consider the set-valued interval functions defined by

$$
\begin{aligned}
& G: B \rightarrow P(Q) \\
& G(X)=\left\{\left[\begin{array}{ll}
0 & k \cdot X \\
0 & k \cdot X
\end{array}\right]: k \in \mathbb{R}\right\},
\end{aligned}
$$

for every $X \in 2 \cdot \Omega_{C}(\mathbb{R})$ and

$$
\begin{aligned}
& F: C \rightarrow P(Q) \\
& F(X)=\left\{\left[\begin{array}{cc}
0 & k \cdot X \\
0 & 0
\end{array}\right]: k \in \mathbb{R}\right\},
\end{aligned}
$$

for every $X \in 4 \cdot \Omega_{C}(\mathbb{R})$. For every $X \in 4 \cdot \Omega_{C}(\mathbb{R}), F(X)$ is a subquasilinear space of $G(X)$. So, $\left(F, 4 \cdot \Omega_{C}(\mathbb{R})\right)$ is a soft subquasilinear space of $\left(G, 2 \cdot \Omega_{C}(\mathbb{R})\right)$. Further, let $(F, D)$ be an another soft set over $Q=M_{2}\left(\Omega_{C}(\mathbb{R})\right)$, where $D=2 \cdot \Omega_{C}(\mathbb{R})$ and

$$
\begin{aligned}
& F: D \rightarrow P(Q) \\
& F(X)=\left\{\left[\begin{array}{cc}
0 & 0 \\
0 & k \cdot X
\end{array}\right]: k \in \mathbb{R}\right\},
\end{aligned}
$$

for every $X \in 2 \cdot \Omega_{C}(\mathbb{R})$. Let $(F, C)+(F, D)=(E, C \times D)$, where $E(X, Y)=F(X)+F(Y)$ for every $(X, Y) \in C \times D$. Then

$$
E(X, Y)=\left\{\left[\begin{array}{ll}
0 & k \cdot X \\
0 & k \cdot X
\end{array}\right]: k \in \mathbb{R}\right\}
$$

is a subquasilinear space of $M_{2}\left(\Omega_{C}(\mathbb{R})\right)$ for every $(X, Y) \in C \times D$. From here, we get $(E, C \times D)$ is a soft quasilinear space over $M_{2}\left(\Omega_{C}(\mathbb{R})\right)$.

Definition 21. Let $(G, B)$ be a non-null soft quasilinear spaces over a quasilinear space $Q$. $(G, B)$ is called proper soft set if the following cases hold:

a) $F_{b}^{(G, B)} \neq \varnothing$ for all $b \in \operatorname{Supp}(G, B)$, 
b) $F_{b_{1}}^{(G, B)} \neq F_{b_{2}}^{(G, B)}$ for every $b_{1}, b_{2} \in \operatorname{Supp}(G, B)$ with $b_{1} \neq b_{2}$.

Otherwise, $(G, B)$ is called improper soft set. If $(G, B)$ is proper soft set then $(G, B)$ is called proper soft quasilinear space.

Clearly, every soft linear space is a proper soft quasilinear space with "=".

Example 22. Let us consider the quasilinear space $\Omega_{C}(\mathbb{R})$. Let $(G, B)$ be a soft set over $\Omega_{C}(\mathbb{R})$, where $B=\left(\Omega_{C}(\mathbb{R})\right)_{r}$ and $G:\left(\Omega_{C}(\mathbb{R})\right)_{r} \rightarrow P\left(\Omega_{C}(\mathbb{R})\right)$ is described by $G(b)=[-b, b]$ for every $b \in\left(\Omega_{C}(\mathbb{R})\right)_{r}$. Clearly, $\left(G,\left(\Omega_{C}(\mathbb{R})\right)_{r}\right)=\left(\Omega_{C}(\mathbb{R})\right)_{d}$. From here, $\left(G,\left(\Omega_{C}(\mathbb{R})\right)_{r}\right)$ is a soft quasilinear space since $\left(\Omega_{C}(\mathbb{R})\right)_{d}$ is a subquasilinear space of $\Omega_{C}(\mathbb{R})$. Further, $\left(G,\left(\Omega_{C}(\mathbb{R})\right)_{r}\right)$ is an improper soft quasilinear space since $\left(\Omega_{C}(\mathbb{R})\right)_{d}$ is an imroper.

Example 23. Let $Q=\Omega_{C}(\mathbb{R})$ be quasilinear space over $\mathbb{R}$ and $(G, B)$ be a soft set over $Q$, where $B=\Omega_{C}(\mathbb{R})$ and $G: \Omega_{C}(\mathbb{R}) \rightarrow P(Q)$ is defined by $G(X)=\{X \cdot k: k \in \mathbb{R}\}$ for every $X \in$ $\Omega_{C}(\mathbb{R}) .\left(G, \Omega_{C}(\mathbb{R})\right)$ is a soft quasilinear space since $G(X)$ is a subquasilinear space of $\Omega_{C}(\mathbb{R})$. Also, $\left(G, \Omega_{C}(\mathbb{R})\right)$ is a proper soft quasilinear space since $\Omega_{C}(\mathbb{R})$ is a proper quasilinear space. If $B=\left(\Omega_{C}(\mathbb{R})\right)_{S}$ and $G:\left(\Omega_{C}(\mathbb{R})\right)_{S} \rightarrow P(Q)$ is defined by $G(X)=\{X \cdot k: k \in \mathbb{R}\}$ for every $X \in Q$. $\left(G,\left(\Omega_{C}(\mathbb{R})\right)_{S}\right)$ is a soft quasilinear space since $G(X)$ is a subquasilinear space of $\Omega_{C}(\mathbb{R})$. But, $\left(G,\left(\Omega_{C}(\mathbb{R})\right)_{S}\right)$ is an improper soft quasilinear space since $\left(\Omega_{C}(\mathbb{R})\right)_{S}$ is an improper quasilinear space.

Example 24. Let $\Omega_{C}\left(\mathbb{R}^{2}\right)$ be the quasilinear space over $\mathbb{R}$ and $(G, B)$ be a soft set over $\Omega_{C}\left(\mathbb{R}^{2}\right)$, where $B=\{\{(m, k)\}: m=0, k \in \mathbb{R}\}$ and $G: B \rightarrow P\left(\Omega_{C}\left(\mathbb{R}^{2}\right)\right)$ is a function defined by

$$
G(X)=\left\{Z \in\left(\Omega_{C}\left(\mathbb{R}^{2}\right)\right)_{S} \cup B: X \subseteq Z \text { for a } X \in B\right\}
$$

Since $G(X)$ is a subquasilinear space of $\Omega_{C}\left(\mathbb{R}^{2}\right)$ for every $X \in \operatorname{Supp}(G, B),(G, B)$ is a soft quasilinear space over $\Omega_{C}\left(\mathbb{R}^{2}\right)$. Further, if we take two different element in $\operatorname{Supp}(G, B)$ such as

$$
\begin{aligned}
& X_{1}=\{\{(m, k)\}: m=0,1 \leq k \leq 2\}, \\
& X_{2}=\{\{(m, k)\}: 0 \leq m \leq 1,1 \leq k \leq 2\},
\end{aligned}
$$

we get 


$$
F_{X_{1}}^{(G, B)}=F_{X_{2}}^{(G, B)}=\{\{(m, k)\}: m=0,1 \leq k \leq 2\} .
$$

Thus, $(G, B)$ is an improper soft quasilinear subspace of proper quasilinear space $\Omega_{C}\left(\mathbb{R}^{2}\right)$.

\section{Soft Quasi Vectors and Soft Normed Quasilinear Spaces}

In this section, we will give the notion of soft normed quasilinear space which is one of the fundamental purposes of the study. Firstly, we will give the notion of soft normed quasilinear space and soft quasi vector and some results related this notions. Later, we present the definition of soft quasilinear operator.

Definition 25. Let $(G, B)$ is a soft quasilinear space of $Q$. A soft element of $Q$ is said to be a soft quasi vector of $(G, B)$. A soft element of the soft $\operatorname{set}(\mathbb{R}, B)$ is said to be a soft scalar.

Example 26. Let us consider the Example 12 and the soft quasilinear space $\left(G,\left(\Omega_{C}(\mathbb{R})\right)_{r}\right)$. Let $\tilde{q}$ is a soft element of $\Omega_{C}(\mathbb{R})$ as the following;

$$
\tilde{q}(r)=[-r, r] \in \Omega_{C}(\mathbb{R}) .
$$

Then $\tilde{q}$ is a soft quasi vectors of $\left(G,\left(\Omega_{C}(\mathbb{R})\right)_{r}\right)$.

A soft quasi vector $\tilde{q}$ in a soft quasilinear space $(G, B)$ is said to be null soft quasi vector if $\tilde{q}(b)=\theta$ for every $b \in B$. The zero element of quasilinear space $Q$ will be denoted by $\Theta$.

Let $\tilde{q}$ and $\tilde{w}$ be soft quasi vectors of $(G, B)$ and $\tilde{k}$ be a soft scalar. Then a partial order relation $\tilde{q} \preccurlyeq \tilde{w}$, the sum $\tilde{q}+\tilde{w}$ of $\tilde{q}, \tilde{w}$, and scalar multiplication $\tilde{k} \cdot \tilde{q}$ are defined by

$$
\begin{aligned}
& q \leqslant \tilde{w} \Leftrightarrow \tilde{q} \tilde{(}(b) \preccurlyeq \tilde{w}(b), \\
& (\tilde{q}+\tilde{w})(b)=\tilde{q}(b)+\tilde{w}(b), \\
& (\tilde{k} \cdot \tilde{q})(b)=\tilde{k}(b) \cdot \tilde{q}(b), \text { for all } b \in B .
\end{aligned}
$$

From here, we have $\tilde{q}+\tilde{w}$ and $\tilde{k} \cdot \tilde{q}$ are soft quasi vectors of $(G, B)$.

Proposition 27. Let $(G, B)$ be a soft quasilinear space over $Q$. Then
a) $\overline{0} \cdot \tilde{q}=\Theta$, for all $\tilde{q} \in(G, B)$,
b) $\tilde{k} \cdot \Theta=\Theta$, for all soft scalar $\tilde{k}$, 
c) $(-\tilde{1}) \cdot \tilde{q}=-\tilde{q}$, for all $\tilde{q} \in(G, B)$.

Proof. The proof is similar to soft linear spaces.

Definition 28. Let $(G, B)$ be a soft quasilinear space of quasilinear space $Q$. Let $\tilde{q}_{1}, \tilde{q}_{2}, \ldots, \tilde{q}_{n} \in(G, B)$ and $\tilde{\alpha}_{1}, \tilde{\alpha}_{2}, \ldots, \tilde{\alpha}_{n} \in(\mathbb{R}, B)$. The element

$$
\tilde{\alpha}_{1} \cdot \tilde{q}_{1}+\tilde{\alpha}_{2} \cdot \tilde{q}_{2}+\cdots+\tilde{\alpha}_{n} \cdot \tilde{q}_{n}=\sum_{k=1}^{n} \tilde{\alpha}_{k} \cdot \tilde{q}_{k}
$$

of $(G, B)$ is said to be quasilinear combination of the soft quasi vectors $\tilde{q}_{1}, \tilde{q}_{2}, \ldots, \tilde{q}_{n}$.

Example 29. Let us consider the soft quasilinear space $\left(G,\left(\Omega_{C}(\mathbb{R})\right)_{r}\right)$ as Example 12. Let $\tilde{q}_{n}=[-n, n] \in \Omega_{C}(\mathbb{R})$ for $n=1,2,3$. Then $\tilde{q}_{1}+\overline{2} \cdot \tilde{q}_{2}, \overline{3} \cdot \tilde{q}_{1}+\tilde{q}_{2}+\tilde{q}_{3}$ are some quasilinear combinations of $\tilde{q}_{1}, \tilde{q}_{2}, \tilde{q}_{3}$.

Definition 30. Let $(G, B)$ be a soft quasilinear space of quasilinear space $Q \cdot\left(\tilde{q}_{k}\right)_{k=1}^{n} \subset$ $(G, B)$ and $\left(\tilde{\alpha}_{k}\right)_{k=1}^{n} \subset(\mathbb{R}, B)$. If

$$
\Theta \preccurlyeq \tilde{\alpha}_{1} \cdot \tilde{q}_{1}+\tilde{\alpha}_{2} \cdot \tilde{q}_{2}+\cdots+\tilde{\alpha}_{n} \cdot \tilde{q}_{n^{\prime}}
$$

implies $\tilde{\alpha}_{1}=\tilde{\alpha}_{2}=\cdots=\tilde{\alpha}_{n}=\overline{0}$, then $\left(\tilde{q}_{k}\right)_{k=1}^{n}$ is said to be quasilinear independent, otherwise $\left(\tilde{q}_{k}\right)_{k=1}^{n}$ is said to be quasilinear dependent.

Example 31. Let us consider again the soft quasilinear space $\left(G,\left(\Omega_{C}(\mathbb{R})\right)_{r}\right)$ as Example 12 and $Z=\left\{\tilde{q}_{n}=[-n, n]: n=1,2,3\right\} \subset(G, B)$ and $\left(\tilde{\alpha}_{k}\right)_{k=1}^{3} \subset(\mathbb{R}, B)$. Then

$$
\Theta \preccurlyeq \tilde{\alpha}_{1} \cdot \tilde{q}_{1}+\tilde{\alpha}_{2} \cdot \tilde{q}_{2}+\tilde{\alpha}_{3} \cdot \tilde{q}_{3}
$$

is satisfied every $\left(\tilde{\alpha}_{k}\right)_{k=1}^{3} \subset(\mathbb{R}, B)$. So, $Z$ is quasilinear dependent in $\left(G,\left(\Omega_{C}(\mathbb{R})\right)_{r}\right)$.

Example 32. Let $I \mathbb{R}^{2}$ be the quasilinear space over $\mathbb{R},(G, I \mathbb{R})$ be a soft set over $I \mathbb{R}^{2}$ and $G: I \mathbb{R} \rightarrow P\left(I \mathbb{R}^{2}\right)$ is function defined by

$$
G(X)=\{(0, X \cdot m): m \in \mathbb{R}\}
$$

for all $(X) \in I \mathbb{R}$. Since $(G, I \mathbb{R})$ is subquasilinear space of $I \mathbb{R}^{2},(G, I \mathbb{R})$ is a soft quasilinear space over $I \mathbb{R}^{2}$. The subset $\left\{\tilde{q}_{n}=(0,[n, n+1]): n=1,2,3\right\} \subset(G, B)$ is quasilinear independent in $(G, B)$ because of 


$$
\Theta \preccurlyeq \tilde{\alpha}_{1} \cdot(0,[1,2])+\tilde{\alpha}_{2} \cdot(0,[2,3])+\tilde{\alpha}_{3} \cdot(0,[3,4])
$$

is satisfied if and only if $\tilde{\alpha}_{1}=\tilde{\alpha}_{2}=\tilde{\alpha}_{3}=\overline{0}$.

The set of all soft quasi vectors over $\tilde{Q}$ will be denoted by $\operatorname{SQV}(\tilde{Q})$.

Theorem 33. The set $\operatorname{SQV}(\tilde{Q})$ is a quasilinear space with the relation "乏̃ "

$$
\tilde{q}_{e_{1}} \tilde{\lessgtr} \tilde{w}_{e_{2}} \Leftrightarrow \tilde{q} \preccurlyeq \tilde{w} \text { and } e_{1} \leq e_{2}
$$

the sum operation

$$
\tilde{q}_{e_{1}}+\tilde{w}_{e_{2}}=(\widetilde{q+w})_{e_{1}+e_{2}}
$$

and the soft real-scalar multiplication

$$
\tilde{\alpha} \cdot \tilde{q}_{e_{1}}=(\widetilde{\alpha \cdot q})_{\alpha e_{1}}
$$

for every $\tilde{q}_{e_{1}}, \tilde{w}_{e_{2}} \in S Q V(\tilde{Q})$ and for every soft real numbers $\tilde{\alpha}$.

Proof. Clearly, $\tilde{q}_{e_{1}} \tilde{\lessgtr} \tilde{q}_{e_{1}}$ for every $\tilde{q}_{e_{1}} \in \operatorname{SQV}(\tilde{Q})$. Let $\tilde{q}_{e_{1}} \tilde{\lessgtr} \tilde{w}_{e_{2}}$ and $\tilde{w}_{e_{2}} \approx \tilde{z}_{e_{3}}$ for every $\tilde{q}_{e_{1}}, \tilde{w}_{e_{2}}, \tilde{z}_{e_{3}} \in S Q V(\tilde{Q})$. Since $\tilde{Q}$ is an absolute soft quasilinear space, we obtain $\tilde{q}_{e_{1}} \preccurlyeq \tilde{z}_{e_{3}}$.

$$
\begin{aligned}
& \tilde{q}_{e_{1}}+\tilde{w}_{e_{2}}=(\widetilde{q+w})_{e_{1}+e_{2}}=(\widetilde{w+q})_{e_{2}+e_{1}}=\tilde{w}_{e_{2}}+\tilde{q}_{e_{1}} . \\
& \tilde{q}_{e_{1}}+\left(\tilde{w}_{e_{2}}+\tilde{z}_{e_{3}}\right)=\tilde{q}_{e_{1}}+(\widetilde{w+z})_{e_{2}+e_{3}}=(q \widetilde{+w}+z)_{e_{1}+e_{2}+e_{3}}=\left(\tilde{q}_{e_{1}}+\tilde{w}_{e_{2}}\right)+\tilde{z}_{e_{3}} .
\end{aligned}
$$

If $\theta \in Q$ is zero vector then $\tilde{q}_{e_{1}}+\theta_{0}=(\widetilde{q+\theta})_{e_{1}+0}=\tilde{q}_{e_{1}}$. So, $\theta_{0}$ is zero vector in $\operatorname{SQV}(\tilde{Q})$.

$$
\begin{aligned}
& \tilde{\alpha} \cdot\left(\tilde{\beta} \cdot \tilde{q}_{e_{1}}\right)=\tilde{\alpha} \cdot(\widetilde{\beta \cdot q})_{\beta e_{1}}=(\tilde{\alpha} \tilde{\beta}) \cdot \tilde{q}_{e_{1}} \cdot \\
& \tilde{\alpha} \cdot\left(\tilde{q}_{e_{1}}+\tilde{w}_{e_{2}}\right)=\tilde{\alpha} \cdot(\widetilde{q+w})_{e_{1}+e_{2}}=(\alpha \cdot \widetilde{q+\alpha} \cdot w)_{\alpha e_{1}+\alpha e_{2}}=\tilde{\alpha} \cdot(\tilde{q})_{e_{1}}+\tilde{\alpha} \cdot \tilde{w}_{e_{2}} \cdot \\
& \tilde{1} \cdot \tilde{q}_{e_{1}}=(\widetilde{1 \cdot q})_{e_{1}}=\tilde{q}_{e_{1}} \cdot \\
& \theta_{0} \cdot \tilde{q}_{e_{1}}=(\widetilde{\theta \cdot q})_{0 . e_{1}}=\theta_{0} . \\
& (\tilde{\alpha}+\tilde{\beta}) \cdot \tilde{q}_{e_{1}}=((\alpha \widetilde{+\beta}) \cdot q)_{(\alpha+\beta) e_{1}} \preccurlyeq(\alpha \cdot \widetilde{q+\beta} \cdot q)_{\alpha e_{1}+\beta e_{1}} \\
& =(\widetilde{\alpha \cdot q})_{\alpha e_{1}}+(\widetilde{\beta \cdot q})_{\beta e_{1}}=\tilde{\alpha} \cdot \tilde{q}_{e_{1}}+\tilde{\beta} \cdot \tilde{q}_{e_{1}} .
\end{aligned}
$$


If $\tilde{q}_{e_{1}} \approx \tilde{w}_{e_{2}}$ and $\tilde{z}_{e_{3}} \tilde{\sim} \tilde{v}_{e_{4}}$, then $\tilde{q} \preccurlyeq \tilde{w}$ and $e_{1} \leq e_{2}$ and $\tilde{z} \preccurlyeq \tilde{v}$ and $e_{3} \leq e_{4}$. From here, we get $\tilde{q}+\tilde{z} \preccurlyeq \tilde{w}+\tilde{v}$ and $e_{1}+e_{3} \leq e_{2}+e_{4}$. This gives $\tilde{q}_{e_{1}}+\tilde{z}_{e_{3}} \approx \tilde{w}_{e_{2}}+\tilde{v}_{e_{4}}$.

If $\tilde{q}_{e_{1}} \tilde{\lessgtr} \tilde{w}_{e_{2}}$, then $\tilde{q} \preccurlyeq \tilde{w}$ and $e_{1} \leq e_{2}$. Thus, we obtain $\tilde{\alpha} \cdot \tilde{q}_{e_{1}} \tilde{\preccurlyeq} \tilde{\alpha} \cdot \tilde{w}_{e_{2}}$ since $\tilde{\alpha} \cdot \tilde{q} \preccurlyeq \tilde{\alpha} \cdot \tilde{w}$ and $\alpha e_{1} \leq \alpha e_{2}$ for every $\tilde{q}_{e_{1}}, \tilde{w}_{e_{2}}, \tilde{z}_{e_{3}}, \tilde{v}_{e_{4}} \in \operatorname{SQV}(\tilde{Q})$ and for every soft real numbers $\tilde{\alpha}, \tilde{\beta}$.

Definition 34. Let $S Q V(\tilde{Q})$ be a soft quasilinear space and $\tilde{N} \subset S Q V(\tilde{Q})$ be a subset. If $\tilde{N}$ is a soft quasilinear space, then $\tilde{N}$ is said to be a soft quasilinear subspace of $\operatorname{SQV}(\tilde{Q})$ and stated by $S Q V(\tilde{N}) \subset S Q V(\tilde{Q})$.

Definition 35. Let $S Q V(\tilde{Q})$ be a soft quasilinear space. Then a mapping $\|\|:. S Q V(\tilde{Q}) \rightarrow$ $\mathbb{R}^{+}(\mathbb{R})$ is said to be a soft norm on the soft quasilinear space $\operatorname{SQV}(\tilde{Q})$, if $\|$.$\| satisfies the$ following conditions:

(SNQ1) $\left\|\tilde{q}_{e}\right\| \tilde{>} \tilde{0}$ if $\tilde{q}_{e} \neq \tilde{\theta}_{0}$ for all $\tilde{q}_{e} \in \operatorname{SQV}(\tilde{Q})$

(SNQ2) $\left\|\tilde{q}_{e_{1}}+\tilde{w}_{e_{2}}\right\| \tilde{\leq}\left\|\tilde{q}_{e_{1}}\right\|+\left\|\tilde{w}_{e_{2}}\right\|$ for all $\tilde{q}_{e_{1}}, \tilde{w}_{e_{2}} \in \operatorname{SQV}(\tilde{Q})$,

(SNQ3) $\left\|\tilde{\alpha} \cdot \tilde{q}_{e_{1}}\right\|=|\tilde{\alpha}|\left\|\tilde{q}_{e_{1}}\right\|$ for every $\tilde{q}_{e_{1}} \in \operatorname{SQV}(\tilde{Q})$ and for every soft scalar $\tilde{\alpha}$,

(SNQ4) if $\tilde{q}_{e_{1}} \tilde{\xi} \tilde{w}_{e_{2}}$, then $\left\|\tilde{q}_{e_{1}}\right\| \tilde{\leq}\left\|\tilde{w}_{e_{2}}\right\|$ for all $\tilde{q}_{e_{1}}, \tilde{w}_{e_{2}} \in \operatorname{SQV}(\tilde{Q})$,

(SNQ5) if for any $\tilde{\epsilon} \tilde{>} \tilde{0}$ there exists an element $\tilde{z}_{\epsilon} \operatorname{SQV}(\tilde{Q})$ such that $\tilde{q}_{e_{1}} \tilde{\xi}_{e_{2}}+\tilde{z}_{\epsilon}$ and $\left\|\tilde{z}_{\epsilon}\right\| \tilde{\leq} \tilde{\epsilon}$ then $\tilde{q}_{e_{1}} \tilde{\lessgtr} \tilde{w}_{e_{2}}$.

The soft quasilinear space $\operatorname{SQV}(\tilde{Q})$ with a soft norm $\|$.$\| on \tilde{Q}$ is said to be a soft normed quasilinear space and is indicated by $(\tilde{Q},\|\|$.$) .$

Example 36. Let $Q$ be a normed quasilinear space. We define $\|\|:. \operatorname{SQV}(\tilde{Q}) \rightarrow \mathbb{R}^{+}(\mathbb{R})$ by $\left\|\tilde{q}_{e}\right\|=|e|+\|q\|_{Q}$. Then $\|\cdot\|$ satisfied first three norms axioms same as. We only show the other axioms. For every $\tilde{q}_{e_{1}}, \tilde{w}_{e_{2}} \in S Q V(\tilde{Q})$ and for every soft real scalar $\tilde{\epsilon}$.

Let $\tilde{q}_{e_{1}} \tilde{\lessgtr} \tilde{w}_{e_{2}}$. We find $q \preccurlyeq_{Q} w$ and $e_{1} \leq e_{2}$ by Theorem 33 . Since $Q$ is normed quasilinear space, we get $\|q\|_{Q} \leq\|w\|_{Q}$. Then $\left\|\tilde{q}_{e_{1}}\right\| \tilde{\leq}\left\|\tilde{w}_{e_{2}}\right\|$. 
Let for any $\tilde{\epsilon} \tilde{>} \tilde{0}$ there exists an element $\tilde{q}_{\epsilon} S Q V(\tilde{Q})$ such that $\tilde{q}_{e_{1}} \tilde{\xi} \tilde{w}_{e_{2}}+\tilde{z}_{\epsilon}$ and $\left\|\tilde{z}_{\epsilon}\right\| \tilde{\leq} \tilde{\epsilon}$. From Theorem 33, we have $q \preccurlyeq_{Q} w+z$ and $e_{1} \leq e_{2}+\epsilon$. On the other hand, we get $|\epsilon|+\|z\|_{Q} \leq \epsilon$ since $\left\|\tilde{z}_{\epsilon}\right\| \tilde{\leq} \tilde{\epsilon}$. This gives $q \preccurlyeq_{Q} w$. Also, $e_{1} \leq e_{2}$. Thus, we obtain $\tilde{q}_{e_{1}} \tilde{\xi} \tilde{w}_{e_{2}}$.

Definition 37. Let $(\tilde{Q},\|\|$.$) be a soft normed quasilinear space. Soft Hausdorff metric or$ soft norm metric on $\tilde{Q}$ is defined by equality

$$
h_{\tilde{Q}}\left(\tilde{q}_{e_{1}}, \tilde{w}_{e_{2}}\right)=\inf \left\{\tilde{r} \geq \tilde{0}: \tilde{q}_{e_{1}} \tilde{\lessgtr} \tilde{w}_{e_{2}}+\tilde{a}_{1}^{r}, \tilde{w}_{e_{2}} \tilde{\sim} \tilde{q}_{e_{1}}+\tilde{a}_{2}^{r},\left\|\tilde{a}_{i}^{r}\right\| \tilde{\leq} \tilde{r}\right\} .
$$

Same as the definition of Hausdorff metric on normed quasilinear space, we obtain $\tilde{q}_{e_{1}} \approx \tilde{w}_{e_{2}}+\left(\tilde{q}_{e_{1}}-\tilde{w}_{e_{2}}\right)$ and $\tilde{w}_{e_{2}} \tilde{\xi} \tilde{q}_{e_{1}}+\left(\tilde{w}_{e_{2}}-\tilde{q}_{e_{1}}\right)$ for every $\tilde{q}_{e_{1}}, \tilde{w}_{e_{2}} \in \operatorname{SQV}(\tilde{Q})$. So,

$$
h_{\tilde{Q}}\left(\tilde{q}_{e_{1}}, \tilde{w}_{e_{2}}\right) \tilde{\leq}\left\|\tilde{q}_{e_{1}}-\tilde{w}_{e_{2}}\right\|
$$

Here, we should note that $h_{\tilde{Q}}\left(\tilde{q}_{e_{1}}, \tilde{w}_{e_{2}}\right)$ may not equal to $\left\|\tilde{q}_{e_{1}}-\tilde{w}_{e_{2}}\right\|$ since $\tilde{Q}$ is a soft quasilinear space.

Proposition 38. Let $(\tilde{Q},\|\|$.$) be a soft normed quasilinear space. The function$ $h_{\tilde{Q}}\left(\tilde{q}_{e_{1}}, \tilde{w}_{e_{2}}\right)$ satisfies all of the soft metric axioms for all $\tilde{x}_{e_{1}}, \tilde{y}_{e_{2}} \in \tilde{Q}$.

Proof. We get, $h_{\tilde{Q}}\left(\tilde{q}_{e_{1}}, \tilde{w}_{e_{2}}\right) \tilde{\geq} \tilde{0}$ from definition of soft Hausdorff metric for all $\tilde{q}_{e_{1}}, \tilde{w}_{e_{2}} \in$ $\tilde{Q}$. If $h_{\tilde{Q}}\left(\tilde{q}_{e_{1}}, \tilde{w}_{e_{2}}\right)=\tilde{0}$, then $\tilde{q}_{e_{1}} \approx \tilde{w}_{e_{2}}$ and $\tilde{w}_{e_{2}} \tilde{\xi} \tilde{q}_{e_{1}}$ since $\tilde{Q}$ is soft normed quasilinear space. Thus, we get $\tilde{q}_{e_{1}}=\tilde{w}_{e_{2}}$ for all $\tilde{q}_{e_{1}}, \tilde{w}_{e_{2}} \in \tilde{Q}$. Conversely, if $\tilde{q}_{e_{1}}=\tilde{w}_{e_{2}}$, then $\tilde{q}_{e_{1}} \approx \tilde{w}_{e_{2}}$ and $\tilde{w}_{e_{2}} \tilde{\xi} \tilde{q}_{e_{1}}$. Hence, we find $h_{\tilde{Q}}\left(\tilde{q}_{e_{1}}, \tilde{w}_{e_{2}}\right)=\tilde{0}$. Clearly, $h_{\tilde{Q}}\left(\tilde{q}_{e_{1}}, \tilde{w}_{e_{2}}\right)=h_{\tilde{Q}}\left(\tilde{w}_{e_{2}}, \tilde{q}_{e_{1}}\right)$. From (SNQ2) and definition of soft Hausdorff metric, we have $h_{\tilde{Q}}\left(\tilde{q}_{e_{1}}, \tilde{z}_{e_{3}}\right) \tilde{\leq} h_{\tilde{Q}}\left(\tilde{q}_{e_{1}}, \tilde{w}_{e_{2}}\right)+$ $h_{\tilde{Q}}\left(\tilde{w}_{e_{2}}, \tilde{z}_{e_{3}}\right)$.

On a soft normed quasilinear space the following conditions always true (here, soft Hausdorff metric $h_{\tilde{Q}}$ induced by soft norm):

$$
h_{\tilde{Q}}\left(\tilde{\alpha} \cdot \tilde{q}_{e_{1}}, \tilde{\alpha} \cdot \tilde{w}_{e_{2}}\right)=\tilde{\alpha} \cdot h_{\tilde{Q}}\left(\tilde{q}_{e_{1}}, \tilde{w}_{e_{2}}\right) \text {, }
$$

for all soft scalar $\tilde{\alpha}$,

$$
h_{\tilde{Q}}\left(\tilde{q}_{e_{1}}+\tilde{w}_{e_{2}}, \tilde{z}_{e_{3}}+\tilde{v}_{e_{4}}\right) \tilde{\leq} h_{\tilde{Q}}\left(\tilde{q}_{e_{1}}, \tilde{z}_{e_{3}}\right)+h_{\tilde{Q}}\left(\tilde{w}_{e_{2}}, \tilde{v}_{e_{4}}\right),
$$




$$
\left\|\tilde{q}_{e_{1}}\right\|=h_{\tilde{Q}}\left(\tilde{q}_{e_{1}}, \tilde{\theta}\right)
$$

Definition 39. A sequence of soft elements $\left\{\tilde{q}_{e_{n}}^{n}\right\}$ in a soft normed quasilinear space $(\tilde{Q},\|\cdot\|)$ is said to be converges to a soft element $\tilde{q}_{e_{0}}^{0}$ if $h_{\tilde{Q}}\left(\tilde{q}_{e_{n}}^{n}, \tilde{q}_{e_{0}}^{0}\right) \rightarrow \tilde{0}$ as $n \rightarrow \infty$.

Definition 40. A sequence of soft elements $\left\{\tilde{q}_{e_{n}}^{n}\right\}$ in a soft normed quasilinear space $(\tilde{Q},\|\|$.$) is said to be a Cauchy sequence if corresponding to every \tilde{\epsilon} \tilde{>} \tilde{0}, \exists m \in \mathbb{N}$ such that $h_{\tilde{Q}}\left(\tilde{q}_{e_{i}}^{i}, \tilde{q}_{e_{j}}^{j}\right) \tilde{\leq} \tilde{\epsilon}$ for all $i, j>m$ i.e. $h_{\tilde{Q}}\left(\tilde{q}_{e_{i}}^{i}, \tilde{q}_{e_{j}}^{j}\right) \rightarrow \tilde{0}$ as $i, j \rightarrow \infty$.

Theorem 41. The operation of algebraic sum and the operation of multiplication by soft real scalars are continuous according to the soft Hausdorff metric.

Proof. Let $\left\{\tilde{q}_{e_{n}}^{n}\right\} \rightarrow \tilde{q}_{e_{0}}^{0}$ and $\left\{\tilde{w}_{e_{n}}^{n}\right\} \rightarrow \tilde{w}_{e_{0}}^{0}$ in a soft normed quasilinear space $(\tilde{Q},\|\|$.$) as$ $n \rightarrow \infty$. Then there is at least one $n_{0}, n_{0}^{\prime} \in \mathbb{N}$ such that

$$
\tilde{q}_{e_{n}}^{n} \tilde{\lessgtr} \tilde{q}_{e_{0}}^{0}+\tilde{a}_{1}^{r}, \tilde{q}_{e_{0}}^{0} \tilde{\lessgtr} \tilde{q}_{e_{n}}^{n}+\tilde{a}_{2}^{r},\left\|\tilde{a}_{i}^{r}\right\| \tilde{\leq} \frac{\tilde{r}}{2}
$$

and

$$
\tilde{w}_{e_{n}}^{n} \tilde{\xi} \tilde{w}_{e_{0}}^{0}+\tilde{b}_{1}^{r}, \quad \tilde{w}_{e_{0}}^{0} \tilde{\lessgtr} \tilde{w}_{e_{n}}^{n}+\tilde{b}_{2}^{r},\left\|\tilde{b_{i}^{r}}\right\| \tilde{\leq} \frac{\tilde{r}}{2},
$$

for every $n \geq n_{0}$ and for every $n \geq n_{0}^{\prime}$, respectively. Since $\tilde{Q}$ is soft normed quasilinear space, we obtain

$$
\tilde{q}_{e_{n}}^{n}+\tilde{w}_{e_{n}}^{n} \tilde{\lessgtr} \tilde{q}_{e_{0}}^{0}+\tilde{w}_{e_{0}}^{0}+\tilde{a}_{1}^{r}+\tilde{b}_{1}^{r}, \tilde{q}_{e_{0}}^{0}+\tilde{w}_{e_{0}}^{0} \tilde{\lessgtr} \tilde{q}_{e_{n}}^{n}+\tilde{w}_{e_{n}}^{n}+\tilde{a}_{2}^{r}+\tilde{b_{2}^{r}},
$$

for every $n \geq n_{0}, n_{0}^{\prime}$. From (NQ3), we get $\left\|\tilde{a_{i}^{r}}+\tilde{b_{i}^{r}}\right\| \leq\left\|\tilde{a_{i}^{r}}\right\|+\left\|\tilde{b_{i}^{r}}\right\| \tilde{\leq} \tilde{r}$. So, $\tilde{q}_{e_{n}}^{n}+\tilde{w}_{e_{n}}^{n} \rightarrow$ $\tilde{q}_{e_{0}}^{0}+\tilde{w}_{e_{0}}^{0}$.

Let $\left\{\tilde{q}_{e_{n}}^{n}\right\} \rightarrow \tilde{q}_{e_{0}}^{0}$ in a soft normed quasilinear space $(\tilde{Q},\|\|$.$) as n \rightarrow \infty$ and $\tilde{\alpha}$ is a soft scalars. Again, there is at least one $n_{0} \in \mathbb{N}$ such that

$$
\tilde{q}_{e_{n}}^{n} \preccurlyeq \tilde{q}_{e_{0}}^{0}+\tilde{a}_{1}^{r}, \tilde{q}_{e_{0}}^{0} \preccurlyeq \tilde{q}_{e_{n}}^{n}+\tilde{a}_{2}^{r},\left\|\tilde{a}_{i}^{r}\right\| \tilde{\leq} \frac{\tilde{r}}{|\tilde{\alpha}|},
$$

for every $n \geq n_{0}$. Since $\tilde{Q}$ is soft normed quasilinear space, we find

$$
\tilde{\alpha} \cdot \tilde{q}_{e_{n}}^{n} \tilde{\Im} \tilde{\alpha} \cdot \tilde{q}_{e_{0}}^{0}+\tilde{\alpha} \cdot \tilde{a}_{1}^{r}, \tilde{\alpha} \cdot \tilde{q}_{e_{0}}^{0} \tilde{\lessgtr} \tilde{q}_{e_{n}}^{n} \cdot \tilde{\alpha}+\tilde{a}_{2}^{r} \cdot \tilde{\alpha},
$$


for every $n \geq n_{0}$. Now, $\left\|\tilde{\alpha} \cdot \tilde{a}_{i}^{r}\right\|=|\tilde{\alpha}|\left\|\tilde{a}_{i}^{r}\right\| \leq \tilde{r}$. Thus, we obtain $\tilde{\alpha} \cdot \tilde{q}_{e_{n}}^{n} \rightarrow \tilde{\alpha} \cdot \tilde{q}_{e_{0}}^{0}$.

Theorem 42. If $\left\{\tilde{q}_{e_{n}}^{n}\right\}$ and $\left\{\tilde{w}_{e_{n}}^{n}\right\}$ are Cauchy sequences in a soft normed quasilinear space $(\tilde{Q},\|\cdot\|)$ then $\left\{\tilde{q}_{e_{n}}^{n}+\tilde{w}_{e_{n}}^{n}\right\}$ is Cauchy sequence in a soft normed quasilinear space $(\tilde{Q},\|\cdot\|)$.

Proof. Let $\left\{\tilde{q}_{e_{n}}^{n}\right\}$ and $\left\{\tilde{w}_{e_{n}}^{n}\right\}$ are Cauchy sequences in a soft normed quasilinear space $(\tilde{Q},\|\|$.$) . Then for every \tilde{r} \tilde{>} \tilde{0}, \exists K_{1}, K_{2} \in \mathbb{N}$ such that

$$
\tilde{q}_{e_{n}}^{n} \tilde{\lessgtr} \tilde{q}_{e_{m}}^{m}+\tilde{a}_{1}^{r}, \tilde{q}_{e_{m}}^{m} \tilde{\lessgtr} \tilde{q}_{e_{n}}^{n}+\tilde{a}_{2}^{r},\left\|\tilde{a}_{i}^{r}\right\| \tilde{\leq} \frac{\tilde{r}}{2}
$$

and

$$
\tilde{w}_{e_{n}}^{n} \tilde{\lessgtr} \tilde{w}_{e_{m}}^{m}+\tilde{b}_{1}^{r}, \tilde{w}_{e_{m}}^{m} \tilde{\lessgtr} \tilde{w}_{e_{n}}^{n}+\tilde{b}_{2}^{r},\left\|\tilde{b}_{i}^{r}\right\| \tilde{\leq} \frac{\tilde{r}}{2^{\prime}}
$$

for all $m, n>K_{1}$ and for all $m, n>K_{2}$, respectively. Since $(\tilde{Q},\|\|$.$) is a soft normed quasilinear$ space, we obtain

$$
\tilde{q}_{e_{n}}^{n}+\tilde{w}_{e_{n}}^{n} \approx \tilde{q}_{e_{m}}^{m}+\tilde{w}_{e_{m}}^{m}+\tilde{a}_{1}^{r}+\tilde{b}_{1}^{r}, \tilde{q}_{e_{m}}^{m}+\tilde{w}_{e_{m}}^{m} \tilde{\lessgtr} \tilde{q}_{e_{n}}^{n}+\tilde{w}_{e_{n}}^{n}+\tilde{a}_{2}^{r}+\tilde{b}_{2}^{r}
$$

Now, $\left\|\tilde{a}_{i}^{r}+\tilde{b_{i}^{r}}\right\| \tilde{\leq}\left\|\tilde{a}_{i}^{r}\right\|+\left\|\tilde{b_{i}^{r}}\right\| \tilde{\leq} \tilde{r}$. Otherwise, if we take $K=\max \left\{K_{1}, K_{2}\right\}$ then

$$
\begin{aligned}
& \tilde{q}_{e_{n}}^{n}+\tilde{w}_{e_{n}}^{n} \tilde{\lessgtr} \tilde{q}_{e_{m}}^{m}+\tilde{w}_{e_{m}}^{m}+\tilde{a}_{1}^{r}+\tilde{b}_{1}^{r}, \\
& \tilde{q}_{e_{m}}^{m}+\tilde{w}_{e_{m}}^{m} \tilde{\sim} \tilde{q}_{e_{n}}^{n}+\tilde{w}_{e_{n}}^{n}+\tilde{a}_{2}^{r}+\tilde{b_{2}^{r}},\left\|\tilde{a_{i}^{r}}+\tilde{b_{i}^{r}}\right\| \tilde{\leq} \tilde{r},
\end{aligned}
$$

for all $m, n>K$. Hence, $\left\{\tilde{q}_{e_{n}}^{n}+\tilde{w}_{e_{n}}^{n}\right\}$ is Cauchy sequence in $\tilde{Q}$.

Definition 43. Let $\lambda: \operatorname{SQV}(\tilde{Q}) \rightarrow S Q V(\tilde{W})$ be a soft mapping. Then $\lambda$ is said to be a soft quasilinear operator if

$$
\begin{aligned}
& \text { (SQO1) } \lambda\left(\tilde{q}_{e}+\tilde{w}_{e^{\prime}}\right) \tilde{\preccurlyeq} \lambda\left(\tilde{q}_{e}\right)+\lambda\left(\tilde{w}_{e^{\prime}}\right), \\
& (\mathrm{SQO} 2) \lambda\left(\tilde{\alpha} \cdot \tilde{q}_{e}\right)=\tilde{\alpha} \cdot \lambda\left(\tilde{q}_{e}\right), \\
& (\mathrm{SQO} 3) \tilde{q}_{e} \tilde{\xi}_{e^{\prime}} \Rightarrow \lambda\left(\tilde{q}_{e}\right) \tilde{\preccurlyeq} \lambda\left(\tilde{w}_{e^{\prime}}\right)
\end{aligned}
$$

for every $\tilde{q}_{e}, \tilde{w}_{e^{\prime}} \in S Q V(\tilde{Q})$ and for all soft scalar $\tilde{\alpha}$. 
Example 44. Let us consider the absolute soft set generated by $\Omega_{C}(\mathbb{R})$ and let us show it by $\left.\Omega_{C} \tilde{(} \mathbb{R}\right)$, i.e., $\left.\left(\Omega_{C} \tilde{(} \mathbb{R}\right)\right)(e)=\Omega_{C}(\mathbb{R})$ for every $e \in B \subseteq \Omega_{C}(\mathbb{R})$. Then $\left.\Omega_{C} \tilde{(} \mathbb{R}\right)$ is absolute soft quasilinear space. For a $\left.\tilde{X} \in \Omega_{C} \tilde{(} \mathbb{R}\right)$ let us define $\|\tilde{X}\|(e)=\left\|\tilde{X}_{e}\right\|_{\Omega_{C}(\mathbb{R})}=\sup _{b \in \tilde{X}_{e}}|b|$ for all $e \in$ $B$. Then $\left.\Omega_{C} \tilde{(} \mathbb{R}\right)$ is a soft normed quasilinear space with $\|\tilde{X}\|(e)=\sup _{b \in \tilde{X}_{e}}|b|$. For $\left.\tilde{X} \in \Omega_{C} \tilde{(} \mathbb{R}\right)$, let us define

$$
\lambda(\tilde{X})(e)=\tilde{2} \cdot \tilde{X}_{e}
$$

Clearly, for every $\left.\left.\tilde{X} \in \Omega_{C} \tilde{(} \mathbb{R}\right), \lambda(\tilde{X})(e) \in \Omega_{C} \tilde{(} \mathbb{R}\right)$ for every $e \in B$. Further,

$$
\lambda(\tilde{X}+\tilde{Y})\left(e+e^{\prime}\right)=\tilde{2} \cdot(\tilde{X}+\tilde{Y})_{e+e^{\prime}}=\lambda(\tilde{X})(e)+\lambda(\tilde{Y})\left(e^{\prime}\right)
$$

for every $\left.\tilde{X}, \tilde{Y} \in \Omega_{C} \tilde{(} \mathbb{R}\right)$.

$$
\lambda(\tilde{\alpha} \cdot \tilde{X})(e)=\tilde{2} \cdot \tilde{\alpha} \cdot \tilde{X}_{e}=\tilde{\alpha} \cdot \lambda\left(\tilde{X}_{e}\right)
$$

for every $\left.\tilde{X}, \tilde{Y} \in \Omega_{C} \tilde{(} \mathbb{R}\right)$ and for every soft scalar $\tilde{\alpha}$. Let $\tilde{X}_{e} \tilde{\lessgtr} \tilde{Y}_{e^{\prime}}$ for every $\tilde{X}, \tilde{Y} \in$ $\left.\Omega_{C} \tilde{(} \mathbb{R}\right)$. Clearly, $\lambda\left(\tilde{X}_{e}\right) \approx \lambda\left(\tilde{Y}_{e^{\prime}}\right)$. So, we obtain $\lambda$ is soft quasilinear operator to $\left.\Omega_{C} \tilde{C} \mathbb{R}\right)$ from $\left.\Omega_{C} \tilde{(} \mathbb{R}\right)$.

Definition 45. The soft quasilinear operator $\lambda: S Q V(\tilde{Q}) \rightarrow S Q V(\tilde{W})$ is said to be soft continuous at $\tilde{q}_{e_{0}}^{0} \in \operatorname{SQV}(\tilde{Q})$, if for every sequence $\tilde{q}_{e_{n}}^{n}$ of soft quasi vectors of $\operatorname{SQV}(\tilde{Q})$ with $\tilde{q}_{e_{n}}^{n} \rightarrow \tilde{q}_{e_{0}}^{0}$ as $n \rightarrow \infty$, we get $\lambda\left(\tilde{q}_{e_{n}}^{n}\right) \rightarrow \lambda\left(\tilde{q}_{e_{0}}^{0}\right)$ as $n \rightarrow \infty$.

Definition 46. The soft quasilinear operator $\lambda: S Q V(\tilde{Q}) \rightarrow S Q V(\tilde{W})$ is said to be soft bounded, if there exists a soft real numbers $\tilde{k}$ such that

$$
\left\|\lambda\left(\tilde{q}_{e}\right)\right\| \tilde{\leq} \tilde{k}\left\|\tilde{q}_{e}\right\|
$$

for all $\tilde{q}_{e} \in \operatorname{SQV}(\tilde{Q})$.

Theorem 47. Let $\lambda: S Q V(\tilde{Q}) \rightarrow S Q V(\tilde{W})$ be a soft quasilinear operator. Then $\lambda$ is said to be soft bounded if and only if it is soft continuous.

Proof. Assume that $\lambda: \operatorname{SQV}(\tilde{Q}) \rightarrow \operatorname{SQV}(\tilde{W})$ is soft bounded. Let the sequence $\left\{\tilde{x}_{e_{n}}^{n}\right\}$ is convergent to the $\left\{\tilde{x}_{e_{0}}^{0}\right\}$. From Definition 39, we have there is at least one $n_{0} \in \mathbb{N}$ such that

$$
\tilde{q}_{e_{n}}^{n} \tilde{\lessgtr} \tilde{q}_{e_{0}}^{0}+\tilde{a}_{1}^{r}, \tilde{q}_{e_{0}}^{0} \tilde{\approx} \tilde{q}_{e_{n}}^{n}+\tilde{a}_{2}^{r},\left\|\tilde{a}_{i}^{r}\right\| \tilde{\leq} \tilde{\tilde{r}}
$$


for every $n \geq n_{0}$. Since $\lambda$ is soft quasilinear operator, we obtain

$$
\lambda\left(\tilde{q}_{e_{n}}^{n}\right) \approx \lambda\left(\tilde{q}_{e_{0}}^{0}\right)+\lambda\left(\tilde{a}_{1}^{r}\right)
$$

and

$$
\lambda\left(\tilde{q}_{e_{0}}^{0}\right) \approx \lambda\left(\tilde{q}_{e_{n}}^{n}\right)+\lambda\left(\tilde{a}_{2}^{r}\right)
$$

On the other hand, because of $\lambda$ is soft bounded, we write

$$
\left\|\lambda\left(\tilde{a_{1}^{r}}\right)\right\| \tilde{\leq} \tilde{k}\left\|\tilde{a_{1}^{r}}\right\| \tilde{\leq} \tilde{r} \text { and }\left\|\lambda\left(\tilde{a_{2}^{r}}\right)\right\| \tilde{\leq} \tilde{k}\left\|\tilde{a_{2}^{r}}\right\| \tilde{\leq} \tilde{r}
$$

So, above three inequality give us the soft bounded quasilinear operator $\lambda$ is continuous.

The other side of the proof is similar to soft linear counterpart.

\section{Conclusion}

In this work, the notion of soft quasilinear space is defined. After, the concept of soft quasi vectors in soft quasilinear spaces is presented as a new structure. Also, some consistent results related with this concept are obtained and supported by new examples. Further, the definitions of soft normed quasilinear space and soft proper quasilinear space are introduced. Continuity and boundedness of soft quasilinear operators have been given and proved some related theorems.

\section{References}

[1] Molodtsov, D., Soft set-theory first results, Computational and Applied Mathematics, 37, 19-31, 1999.

[2] Das, S., Samanta, S.K., On soft metric spaces, Journal of Fuzzy Mathematics, 21, 707734, 2013.

[3] Das, S., Samanta, S.K., Soft real sets, soft real numbers and their properties, Journal of Fuzzy Mathematics, 20 (3), 551-576, 2012.

[4] Das, S., Majumdar, P., Samanta, K., On soft linear spaces and soft normed linear spaces, Annals of Fuzzy Mathematics and Informatics, 9(1), 91-109, 2015.

[5] Das, S., Samanta, S.K., Soft linear operators in soft normed linear spaces, Annals of Fuzzy Mathematics and Informatics, 6(2), 295-314, 2013.

[6] Aseev, S.M., Quasilinear operators and their application in the theory of multivalued mappings, Proceedings of the Steklov Institute of Mathematics, 2, 23-52, 1986.

[7] Yılmaz, Y., Çakan, S., Aytekin, Ş., Topological quasilinear spaces, Abstract and Applied Analysis, (2012), 951374, 2012.

[8] Çakan, S., Yılmaz, Y., Normed proper quasilinear spaces, Journal of Nonlinear Sciences and Applications, 8, 816-836, 2015. 
[9] Y1lmaz, Y., Bozkurt, H., Çakan, S., On orthonormal sets in inner product quasilinear spaces, Creative Mathematics and Informatics, 25(2), 237-247, 2016.

[10] Levent, H., Yilmaz, Y., Hahn-Banach extension theorem for interval-valued functions and existence of quasilinear functionals, New Trends in Mathematical Sciences, 6(2), 19-28, 2018.

[11] Levent, H., Yilmaz, Y., Translation, modulation and dilation systems set-valued signal processing, Carpathian Mathematical Publications, 10(1), 143-164, 2018.

[12] Maji, P.K., Biswas, R., Roy, A.R., Soft set theory, Computational and Applied Mathematics, 45, 555-562, 2003.

[13] Feng, F., Jun, Y. B., Zhao, X., Soft semiring, Computational and Applied Mathematics, 56, 2621-2628, 2008.

[14] Das, S., Samanta, S.K., Soft metric, Annals of Fuzzy Mathematics and Informatics, 6(1), 77-94, 2013.

[15] Bayramov, S., Gündüz (Aras), C., Soft locally compact and soft paracompact spaces, Journal of Mathematics and System Science, 3, 122-130, 2013.

[16] Yazar, M.İ., Bilgin, T., Bayramov, S., Gündüz, Ç., A new view on soft normed spaces, International Mathematical Forum, 9(24), 1149-1159, 2014. 